\title{
Conhecimento, prática e percepção sobre o gerenciamento de resíduos de serviços de saúde em estabelecimentos médicos veterinários de Salvador, Bahia
}

\author{
Knowledge, practice and perception about healthcare waste management in veterinary \\ clinics in Salvador, Bahia
}

\author{
REIS, Mariangela Andrade ${ }^{1 *}$; RANGEL-S, Maria Ligia ${ }^{2}$; MATTOS, Camila \\ Maria de ${ }^{1}$; FRANKE, Carlos Roberto ${ }^{1}$
}

\begin{abstract}
${ }^{1}$ Universidade Federal da Bahia, Escola de Medicina Veterinária e Zootecnia, Salvador, Bahia, Brasil.
${ }^{2}$ Universidade Federal da Bahia, Instituto de Saúde Coletiva, Salvador, Bahia, Brasil.

*Endereço para correspondência: mari.andreis@gmail.com
\end{abstract}

\section{RESUMO}

Objetivou-se avaliar os estabelecimentos veterinários e prestadores de serviços clínicos da cidade de Salvador - Bahia, quanto ao cumprimento da legislação referente ao gerenciamento de resíduos de serviços de saúde por meio da análise da prática, do conhecimento, e da percepção dos responsáveis técnicos sobre o tema. Entre setembro de 2010 e março de 2011, 36 clínicas e consultórios veterinários foram visitados e seus respectivos responsáveis técnicos entrevistados sobre o conhecimento, a prática e a percepção sobre o tema. Desses, $30,6 \%$ conheciam a legislação vigente, $2,8 \%$ implantaram o plano de gerenciamento de resíduos de serviços de saúde, $19,4 \%$ tinham contrato com empresa de coleta especial e $88,9 \%$ atribuíam riscos aos resíduos de serviços de saúde, além de outros aspectos que foram investigados. Os resultados mostraram que embora os responsáveis técnicos desconhecessem a resolução da Agencia Nacional de Vigilância Sanitária, eles tinham consciência dos riscos associados às práticas inadequadas de gerenciamento dos resíduos de serviços de saúde. $\mathrm{O}$ estudo revelou a necessidade de se perceber e corrigir as lacunas na formação profissional; na percepção dos riscos representados pelos resíduos de serviços de saúde; no diálogo entre as instituições e o profissional e na formulação de critérios que adéquem a legislação pertinente às características e à escala da geração de resíduos de serviços de saúde nos estabelecimentos médicos veterinários.

Palavras-chave: ANVISA, clinica veterinária, legislação sanitária

\section{SUMMARY}

The aim of this study was to evaluate the veterinary establishments, providers of clinical services in the city of Salvador, Bahia, regarding compliance with laws relating to waste health management, as well as assess the practice, knowledge and perception on the subject. From September 2010 to March 2011, 36 veterinary establishments were visited and their technical managers interviewed about knowledge, practice and perception on the subject. Of these, $30.6 \%$ know the current law, $2.8 \%$ have implemented the plan of waste management of health services, $19.4 \%$ has a contract with the company of collects waste and $88.9 \%$ believes that the waste health services represent some kind of risks. Other aspects were investigated too. The results show that although the technical managers unfamiliar with the resolution of the National Sanitary Surveillance Agency, they are aware of the risks associated with inappropriate practices of waste management of health services. The study revealed the need to understand and correct the gaps in vocational training in the perception of the risks posed by the waste of health services; dialogue between institutions and professional and the formulating criteria that suit the requirements of the relevant legislation and the scale of generation of waste health services in establishments veterinarians.

Keywords: Health legislation, Veterinary legislation, Veterinary medicine 


\section{INTRODUÇÃO}

As condições precárias do gerenciamento dos resíduos no Brasil oferecem riscos tanto à saúde pública (GARCIA \& ZANETTI-RAMOS, 2004) à saúde ocupacional (BARROS et al, 2010). No Brasil a Agência Nacional de Vigilância Sanitária - ANVISA, por meio da RDC 306/04 e o Conselho Nacional do Meio Ambiente CONAMA, pela Resolução CONAMA $358 / 05$, regulamentaram o gerenciamento dos resíduos de serviços de saúde. Ambos os documentos atribuem ao gerador a responsabilidade de providenciar $\mathrm{o}$ adequado gerenciamento dos seus resíduos (BRASIL, 2004; BRASIL, 2005).

A Medicina Veterinária está contemplada na RDC 306/04 e no CONAMA 358/05 como "serviço relacionado com o atendimento de saúde animal”. Segundo o Conselho Federal de Medicina Veterinária é do responsável técnico a atribuição de garantir o cumprimento da RDC 306/04 e do CONAMA 358/05 (CRMV/BA, 2007).

Segundo a legislação, todos os geradores de resíduos de serviços de saúde necessitam executar um Plano de Gerenciamento de Resíduos de Serviços de Saúde que descreva os procedimentos relativos ao manejo de resíduos gerados nas suas dependências (BRASIL, 2004; BRASIL, 2005). Fica determinado ainda que os estabelecimentos precisam contar com um abrigo externo para resíduos; realizar a segregação dos resíduos em grupos A (infectantes), B(químicos), D (comuns) e E (perfuro cortantes); acondicionar os resíduos em recipientes próprios; e providenciar a destinação adequada (BRASIL, 2004). O cumprimento adequado dessas orientações são fundamentais para a redução do volume de resíduos gerados e para a minimização dos riscos, elevando a qualidade e eficiência dos serviços prestados pelo estabelecimento de saúde (SALLES et al, 2009) .

Em Salvador, a coleta e destinação dos resíduos de serviços de saúde era realizada pela Empresa Urbana de Limpeza de Salvador (LIMPURB). Desde 2006, o Poder Público Municipal repassou para o gerador dos resíduos essa responsabilidade e empresas particulares de coleta e tratamento passaram a oferecer estes serviços aos estabelecimentos de saúde.

Embora a legislação sobre resíduos na área de saúde date de 2004 e 2005, o médico veterinário parece ter pouco conhecimento sobre o conteúdo dessas leis. Objetivou-se nesse estudo avaliar o gerenciamento de resíduos de serviços de saúde na área da medicina veterinária abordando a prática, o conhecimento e a percepção sobre $\mathrm{o}$ tema em estabelecimentos prestadores de serviços veterinários em Salvador , Bahia.

\section{MATERIAL E MÉTODO}

A identificação de estabelecimentos veterinários de Salvador - Bahia foi realizada por meio de busca nas listas telefônicas municipais editadas pela Editel (2009-2010) e Telelista (2010), uma vez que não possível ter acesso às listas oficiais dos órgãos responsáveis. Foi elaborado um pré-roteiro de entrevista baseado na revisão bibliográfica sobre o tema objetivandose avaliar o conhecimento dos entrevistados acerca das normas legais de gerenciamento de resíduos, a adequação do manejo interno e analisar a percepção do profissional sobre o gerenciamento dos resíduos de serviços 
de saúde. As visitas aos estabelecimentos ocorreram no período de setembro de 2010 a março de 2011.

Dos 72 estabelecimentos identificados, sete participaram da primeira fase do experimento (entrevistas experimentais a partir do pré-roteiro para adequação da ferramenta metodológica), e 36 da segunda fase (entrevistas a partir do roteiro definitivo semiestruturado), sendo $29(80,6 \%)$ consultórios, e sete $(19,4 \%)$ clínicas. Foram excluídos 29 estabelecimentos pelos seguintes motivos: os responsáveis técnicos optaram em não participar da entrevista, por não haver um responsável técnico no estabelecimento, ou insucesso nas tentativas de contatar o mesmo. As entrevistas foram realizadas de pela pesquisadora e por uma auxiliar, gravadas e posteriormente transcritas para avaliação seguindo as seguintes etapas (BAUER, 2002; NOGUEIRAMARTINS \& BOGUS, 2004):

Codificação - Cada entrevista foi minuciosamente estudada, foram anotadas palavras-chaves e feito um resumo das ideias expostas.

Pdronização - As palavras-chaves e o perfil dos resumos foram agrupados por padrões formando as seguintes categorias: 1) conhecimento teórico legal: referente ao conhecimento sobre a RDC 306/04 da ANVISA; 2) prática do gerenciamento no estabelecimento: referente aos procedimentos adotados no estabelecimento; 3) percepção dos riscos associados aos resíduos gerados por estabelecimentos veterinários: referente às opiniões dos responsáveis técnicos sobre os riscos representados pelos resíduos de serviços de saúde; 4) percepção das causas da problemática dos resíduos de serviços de saúde em Salvador: referente às opiniões dos responsáveis técnicos sobre os fatores que permeiam a questão do gerenciamento de resíduos em estabelecimentos veterinários de Salvador; 5) percepção do retorno mercadológico: referente à interferência do gerenciamento de resíduos de serviços na imagem do estabelecimento e predileção do cliente .

Categorização - Todas as entrevistas foram reavaliadas para categorização das respostas;

Subcategorização - As categorias foram subdividas a fim de facilitar $o$ entendimento, apresentação e interpretação dos dados. A análise estatística realizada foi a descrição de frequências.

$\mathrm{Na}$ categoria 1: conhecimento teórico legal, as respostas foram divididas em quatro subcategorias: a) Conheciam a legislação que trata dos resíduos de serviços de saúde: b) Sabiam o que é um plano de gerenciamento de resíduos de serviços de saúde; c) Informados sobre o correto manejo dos resíduos de serviços de saúde durante a formação profissional; d) Conheciam a classificação dos resíduos de serviços de saúde.

$\mathrm{Na}$ Categoria 2: prática do gerenciamento no estabelecimento, as respostas foram divididas em cinco subcategorias: a) Utilizavam coletor de perfurocortantes; b) Segregavam os resíduos dos grupos A e D; c) Possuíam abrigo externo para resíduos; d) Mantinham contrato com empresa de coleta especial; e) Implementaram o plano de gerenciamento de resíduos de serviços de saúde. Optou-se por não abordar o gerenciamento do Grupo B (químicos) nos resultados da pesquisa uma vez que a maioria dos estabelecimentos declarou não gerar resíduos dessa natureza.

Na categoria 3: Percepção dos Riscos associados aos resíduos gerados por estabelecimentos veterinários as respostas foram divididas em sete subcategorias: a) Saúde Pública (propagação de doenças infecciosas); b) Meio Ambiente 
(contaminação dos solos e água); c) Acidentes com perfurocortantes; d) Saúde Ocupacional: (Saúde e integridade física dos catadores de lixo e garis); e) Nenhum risco associado; f) Reutilização de perfurocortantes (Reutilização de seringas e agulhas descartados de forma inadequada por usuários de drogas); g) Saúde Animal (Risco para saúde dos animais que transitam pelas áreas de descarte).

Na categoria 4: Percepção das Causas da problemática dos resíduos de serviços de saúde em Salvador, as respostas foram divididas em cinco subcategorias: a) Falha dos cursos superiores e conselhos de classe; b) Falta de fiscalização; c) Custos da coleta especial; d) Ausência de coleta municipal; e) Desinteresse dos profissionais.

$\mathrm{Na}$ categoria 5: percepção do retorno mercadológico, apenas duas subcategorias foram geradas: a) Indiferença: $O$ cliente não se importa com o manejo e destinação dos resíduos na clinica e, como consequência, não há retorno financeiro para $o$ estabelecimento; b) Fidelidade do cliente: Existe a preocupação do cliente e a predileção ou fidelidade por estabelecimentos que agem de maneira correta com seus resíduos, trazendo assim um retorno financeiro.

O projeto foi submetido e aprovado pelo Comitê de Ética em Pesquisa do Instituto de Saúde Coletiva/ISC da Universidade Federal da Bahia

\section{RESULTADOS E DISCUSSÃO}

Os resultados obtidos referentes à Categoria 1 (Figura1) mostraram que $69,4 \%$ dos responsáveis técnicos entrevistados não conheciam a RDC 306/04, e, em consequência, desconheciam também o plano de gerenciamento de resíduos de serviços de saúde $(72,2 \%)$ e os critérios de classificação dos resíduos de serviços de saúde $(86,1 \%)$, o que os impossibilitaria a elaboração de procedimentos corretos de manuseio, segregação e destinação dos resíduos de serviços de saúde gerados.

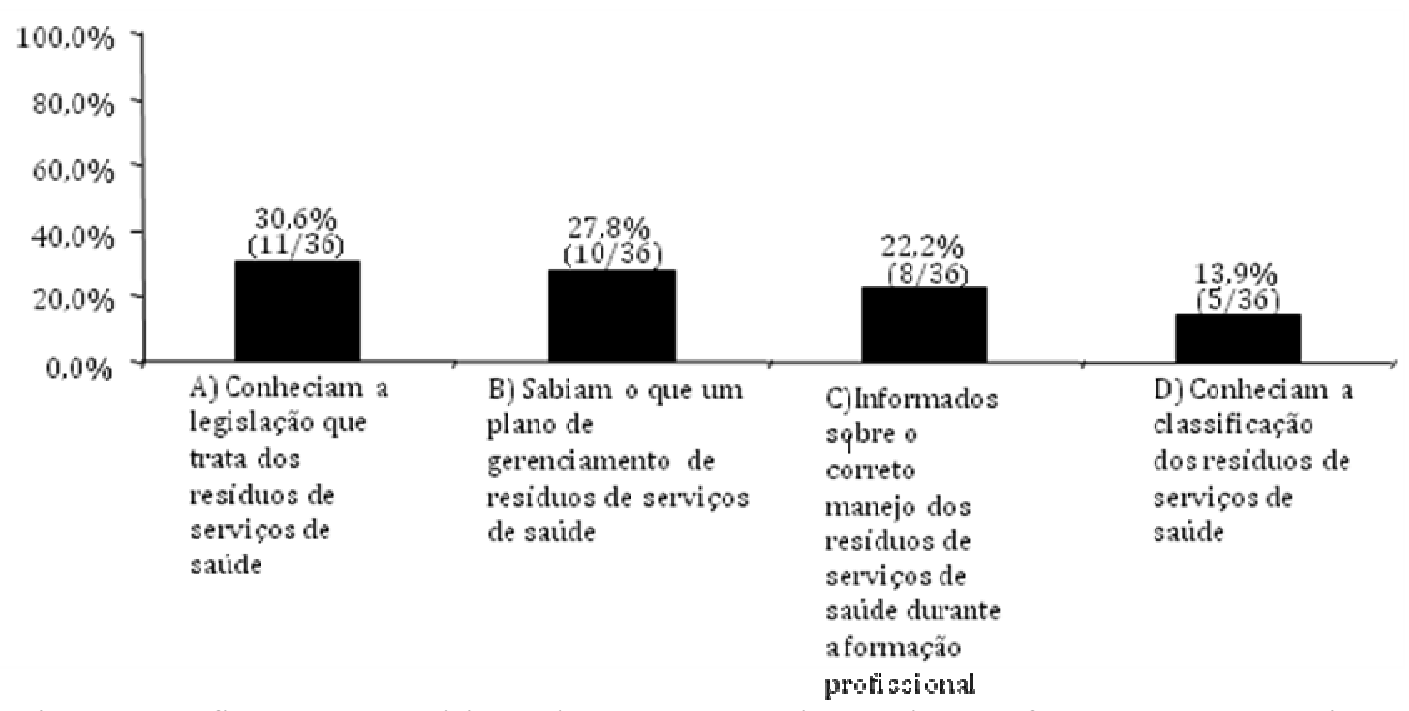

Figura 1. Afirmações positivas dos responsáveis técnicos referentes à Categoria 1, conhecimento teórico legal, divididas em quatro subcategorias 
Um dos fatores que contribuiu para o desconhecimento da legislação vigente é que apenas $22,2 \%$ dos entrevistados tiveram contato com o conteúdo referente ao gerenciamento de resíduos de serviços de saúde na sua formação profissional, seja no curso de graduação ou no curso de formação de responsáveis técnicos oferecido pelo conselho de classe regional. Essa constatação está em acordo com as observações feitas por Correa et al. (2005), que enfatizaram que a percepção da falha na formação profissional ajuda a compreender a presente desinformação sobre os riscos representados pelos resíduos de serviços de saúde ao meio ambiente e à saúde pública. É fato que apenas em 2010, tanto o curso de Medicina Veterinária da Universidade Federal da Bahia, como o curso de formação de responsabilidade técnica do conselho de classe oferecido na Bahia, incluíram o conteúdo referente aos resíduos de serviços de saúde nos seus currículos, no entanto o desinteresse pela busca de informações também pode ter contribuído para o desconhecimento da legislação por parte dos profissionais. Da mesma forma Gonçalves et al. (2011) afirmaram que são pontos básicos para as mudanças em relação ao manejo dos resíduos de serviços de saúde a busca ativa de informações e a observações de normas regulamentadoras, além da qualificação de recursos humanos.

Como resultado, na Categoria 2 notouse que uma ampla parcela dos resíduos de serviços de saúde era acondicionada e descartada inadequadamente (Figura 2).

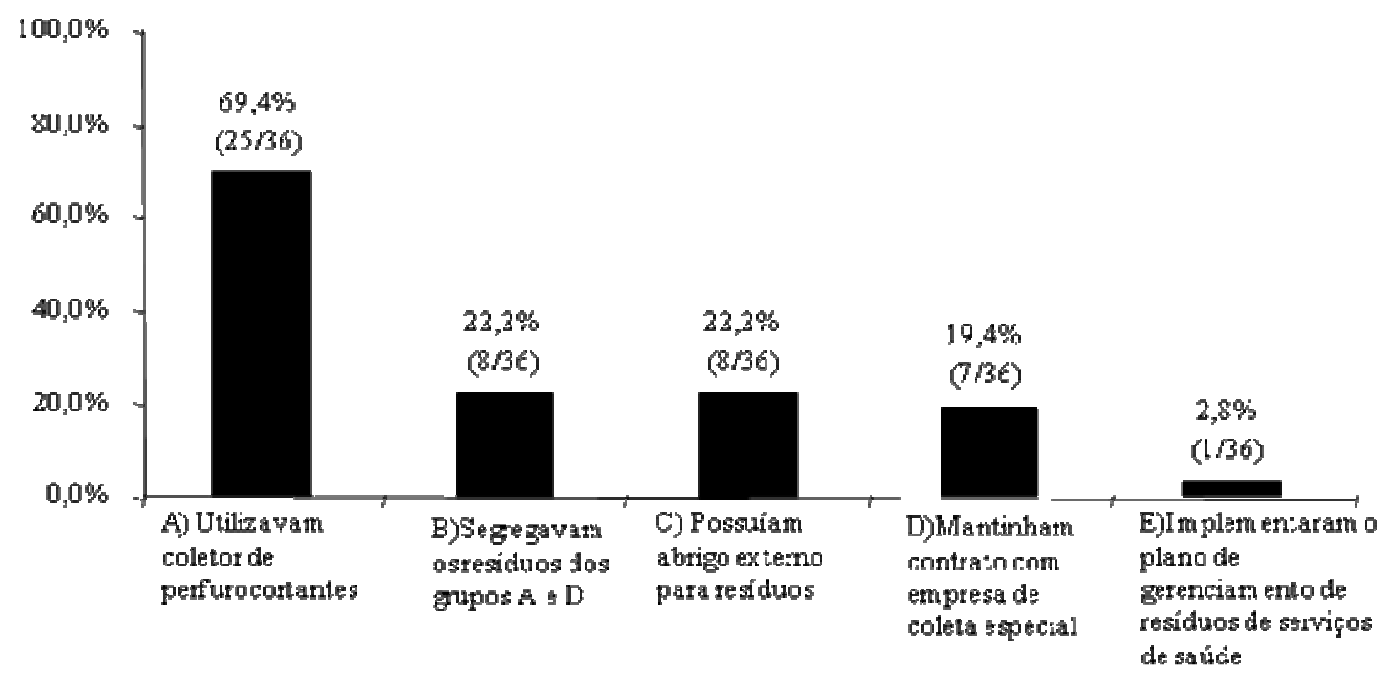

Figura 2. Afirmações positivas dos responsáveis técnicos referentes à Categoria 2, prática do gerenciamento no estabelecimento, divididas em cinco subcategorias

Além disso, apenas um estabelecimento havia implantado o plano de gerenciamento de resíduos de serviços de saúde por exigência da prefeitura de Salvador, com a qual o estabelecimento mantinha um convenio de castração de cães e gatos. Para Garcia \& ZanetteRamos (2004) os pequenos geradores de resíduos de serviços de saúde não contam com grau de consciência e de conhecimentos sobre o gerenciamento de resíduos como os grandes geradores, 
e também não possuem infraestrutura para realiza-lo adequadamente. Esse não é um problema exclusivo dos estabelecimentos veterinários de Salvador. Pilger \& Schenato (2008) relataram um quadro semelhante em um hospital veterinário no Rio Grande do Sul, no qual foram identificadas falhas na segregação dos resíduos. Em hospital veterinário do Paraná, RoederFerrarie et al. (2008) relataram ausência de segregação e acondicionamento dos resíduos conforme exigência da ANVISA. Em Belo Horizonte-MG, 98,1\% dos estabelecimentos odontológicos não separavam os resíduos infectantes dos demais, $50 \%$ não possuíam abrigo externo para resíduos, $100 \%$ utilizava coletor específico para resíduos perfurocortantes, apenas $9 \%$ tinha contrato com empresa de coleta especial e nenhum possuía um plano de gerenciamento de resíduos de serviços de saúde (NAZAR et al., 2005). Salles et al. (2009) avaliaram 13 estabelecimentos de atendimentos à saúde humana, dos quais apenas um acondicionava separadamente os resíduos dos grupos A e $\mathrm{D}$, seis segregavam resíduos do grupo E, e apenas 4 dispunham de abrigo externo para resíduos.

A constatação de que a maioria dos estabelecimentos médicos veterinários avaliados não dispunham de um plano de gerenciamento de resíduos de serviços de saúde (Figura 2), o qual constitui documento obrigatório à concessão do alvará de funcionamento do estabelecimento por parte da vigilância sanitária municipal, impõe uma reflexão sobre a atuação desse órgão, em consonância com as exigências constantes na RDC 306/04 e na Referência Técnica para o Funcionamento dos Serviços Veterinários, ambos emitidos pela ANVISA, mormente no tocante à RDC 306/04 que atribui às vigilâncias sanitárias estaduais e municipais a competência de divulgar, orientar e fiscalizar o cumprimento da referida resolução (BRASIL, 2004; BRASIL, 2010). Vasconcellos et al. (2006) relataram situação parecida em Campina Grande - PB, onde , segundo os autores "As ações políticoadministrativas na área em questão têm se revelado ineficazes ou inexistentes, o que faz com que a descrença da população nas soluções técnicas inviabilizem qualquer ação prática".

Além da desinformação sobre a legislação vigente (Figura 1) a baixa adesão às práticas seguras de gerenciamento dos resíduos de serviços de saúde foi justificada pelos entrevistados, em parte, pelo elevado custo do serviço de coleta especial $(38,9$ $\%$ e pela falta de fiscalização da vigilância sanitária (44,4\%) (Figura 3).

Em Salvador, além de insuficiente o número de empresas privadas de coleta especial, o valor do serviço cobrado torna-se oneroso, especialmente no tocante aos consultórios médicos veterinários (em nosso estudo perfazem $80,6 \%$ do total), os quais, pelo limitado leque de serviços que prestam, geram baixa quantidade diária de resíduos de serviços de saúde e, geralmente, apresentam reduzido movimento de caixa, tornando difícil o atendimento à resolução. Para Rezende (2006) os geradores de resíduos assumem uma condição de vulnerabilidade diante da RDC 306/04, tornando-se dependentes das condições e preços que as empresas de coleta especial estabelecem pelos seus serviços. Em nosso estudo 18\% dos entrevistados apontaram como uma das causa da problemática do gerenciamento de resíduos veterinários em Salvador a ausência da coleta municipal (Figura 3). No entanto é importante frisar que um serviço de coleta municipal gratuito para resíduos 
de saúde não desobrigaria os geradores de elaborarem um plano de gerenciamento, uma vez que a segregação e o tratamento interno dos resíduos de serviços de saúde devem acontecer na fonte, e são indispensáveis para garantir a segurança dos funcionários do estabelecimento e do serviço de coleta. Sobre isso, Rezende (2006) enfatizou que a questão dos resíduos de serviços de saúde não pode ficar centrada apenas no cumprimento das legislações, no que diz respeito ao contrato com uma empresa de coleta especial, ela implica em mudança de conduta, por parte do gerador, quanto à execução de todos os passos do gerenciamento dos resíduos de serviços de saúde .

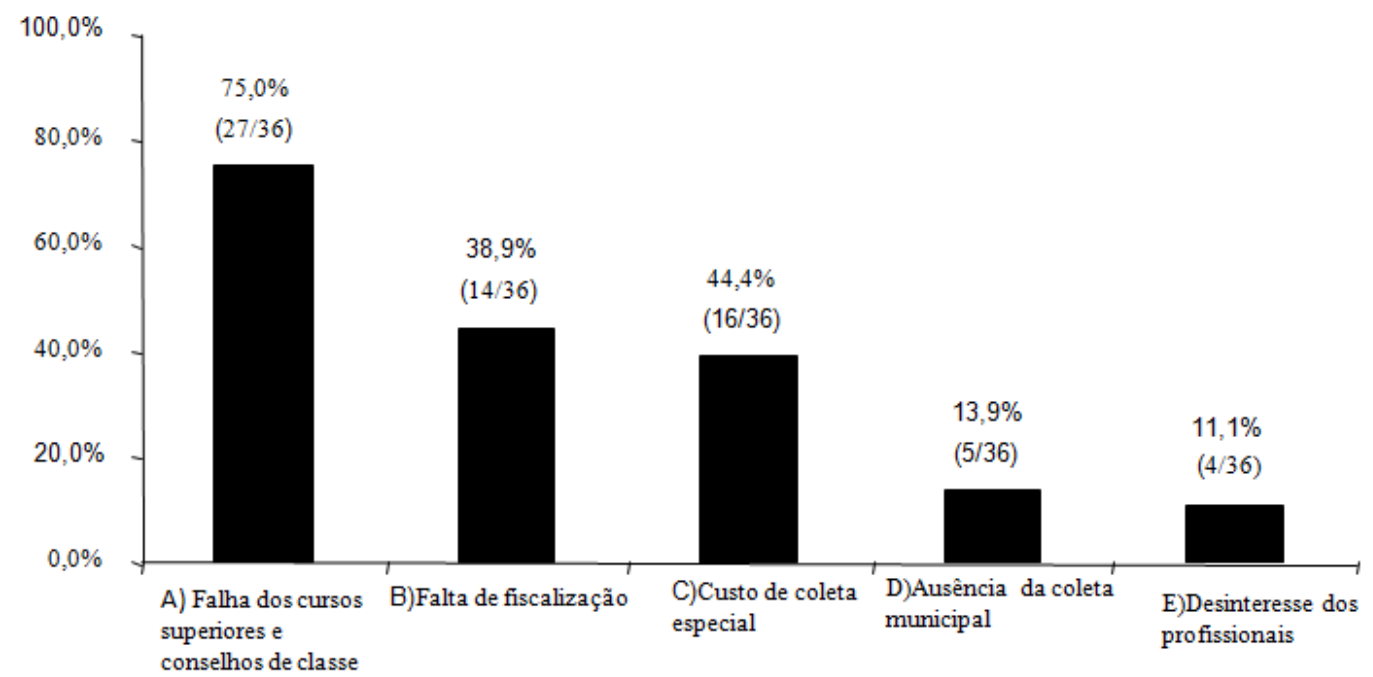

Figura 3. Afirmações dos responsáveis técnicos referentes à Categoria 4, Percepção das Causas da problemática dos resíduos de serviços de saúde em Salvador divididas em cinco subcategorias

De acordo com os dados obtidos na Categoria 3 ( Figura 4), 88,9\% dos responsáveis técnicos demonstraram conhecimento sobre riscos potenciais representados pelos resíduos de serviços de saúde gerados nos estabelecimentos. As afirmações feitas pelos entrevistados estão de acordo com a literatura e sugerem que, apesar da desinformação teórica, a maioria tinha conhecimento dos riscos associados aos resíduos de serviços de saúde. A OMS (WHO, 2004) estimou que em 2000, as injeções com seringas contaminadas causaram 21 milhões de infecções pelo vírus da hepatite B (HBV), dois milhões pelo vírus da hepatite $\mathrm{C}(\mathrm{HCV})$, e pelo menos 260 mil novas infecções pelo HIV. Patógenos como Staphylococcus coagulase, Citrobacter sp.; Providencia sp.; Klebsiella sp.; Proteus sp.; Escherichia coli; Salmonella sp. e Shigella $s p$. foram isoladas em amostras de chorume proveniente de resíduos de serviços de saúde descartados em aterro sanitário (NASCIMENTO et al. , 2004). Sobre o risco ambiental Mbongwe et al. (2008) enfatizaram que os resíduos de serviços de saúde não contêm apenas resíduos infectantes, mas também produtos químicos tóxicos e metais pesados que oferecem risco ambiental, o 
que no Brasil, segundo Bellan et al. (2012) é agravada pela falta de tratamento de esgoto. Sobre o risco ocupacional Barros et al. (2010) relataram que $87,5 \%$ dos trabalhadores de coleta externa de resíduos de serviços de saúde entrevistados por eles já sofreram alguma injúria durante a manipulação desses resíduos. Para Gouveia (2012) os resíduos urbanos representam riscos à saúde para os profissionais mais diretamente envolvidos no manejo, como catadores e trabalhadores da coleta de lixo, os quais muitas vezes não contam com medidas mínimas de prevenção e segurança ocupacional. Pechansky et al. (2000) relataram que 53\% dos usuários de cocaína utilizam agulhas e seringas usadas previamente, o que reforça a indicação de risco associado ao descarte inadequado dos resíduos de serviços de saúde. Vale ressaltar que apenas 11,5\% dos responsáveis técnicos afirmaram acreditar que os resíduos gerados nos seus estabelecimentos não constituem risco, uma vez que os animais atendidos são, na sua maioria, de classe média ou alta, vacinados e avaliados por um medico veterinário regularmente. Segundo a OMS, embora grande parte dos resíduos de serviços de saúde seja de fato inofensiva, a presença de resíduos perigosos, ainda que em pequena quantidade, não pode ser ignorada (WHO, 2004). Em Salvador doenças infecciosas de caráter zoonótico como a brucelose canina (CAVALCANTI et al, 2006), a leishmaniose visceral (BARBOZA et al, 2009), toxoplasmose ( BARBOSA et al, 2003), e doenças parasitarias caninas, também de importancia zoonótica, como Ancylostoma sp.; Toxocara sp.; Toxascaris, Taeniidae, Trichuris e Ascaris (SANTOS et al, 2006) foram identificadas em animais de diversas áreas e submetidos a diferentes tipos de manejo, o que sugere que mesmo estando aparentemente em boas condições qualquer animal está suceptivél à enfermidades podendo se tornar uma fonte de infecção.

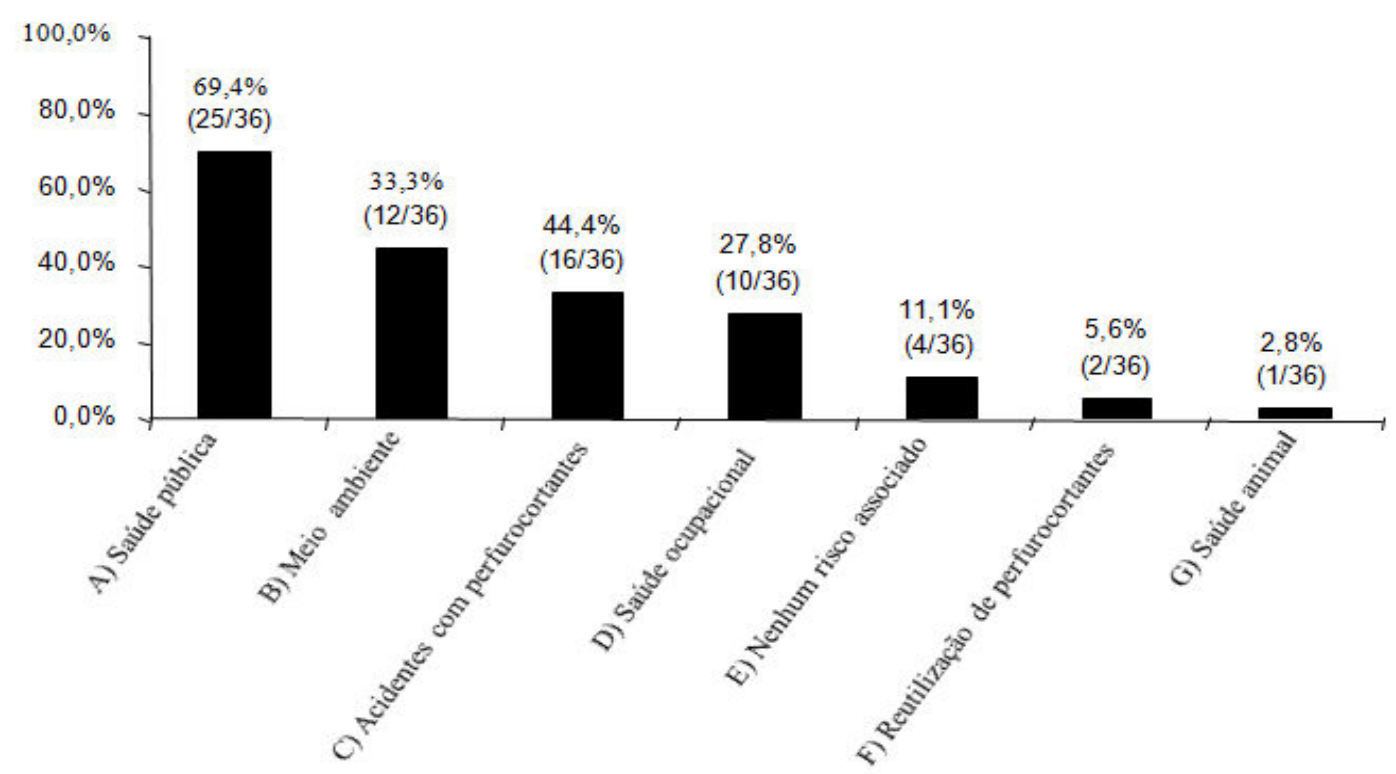

Figura 4.Afirmações positivas dos responsáveis técnicos referentes à Categoria 3, Percepção dos riscos associados aos resíduos gerados por estabelecimentos veterinários, divididas em sete subcategorias 
A constatação de que práticas inadequadas estavam sendo executadas apesar da consciência dos riscos que representam sugere que, embora a alegação de desconhecimento tenha sido apontada por $75 \%$ dos responsáveis técnicos como sendo a principal causa do manejo inapropriado dos resíduos, é possível que os outros fatores citados na Categoria 4 (Figura 3) como: falta de fiscalização e custos dos serviços de coleta, estivessem sendo determinantes na falta de motivação desses profissionais em buscarem informações qualificadas sobre o gerenciamento dos resíduos de serviços de saúde. Reflexo disso é que apenas $11,1 \%$ dos entrevistados atribuíram à falta de interesse profissional ao desconhecimento das legislações referentes aos resíduos de serviços de saúde.

$\mathrm{Na}$ categoria 5, Percepção do retorno mercadológico, a subcategoria "A" (Indiferença) foi mencionada por 58,3\% dos entrevistados e a subcategoria "B" (Fidelidade do cliente) foi mencionada por $38,9 \%$ dos entrevistados. As respostas obtidas na Categoria 5, evidenciaram que ainda é reduzido o número de profissionais $(38,9 \%)$ que acreditam que a adoção de boas práticas de manejo de resíduos no estabelecimento repercuta positivamente. A questão da percepção do cliente está diretamente relacionada ao seu nível de informação sobre os riscos associados aos resíduos de serviços de saúde e à importância do correto gerenciamento. Segundo Silva (2005) para a conscientização da população sobre a importância do manejo adequado dos resíduos de serviços de saúde é necessário a formulação de ações integradas entre diversos segmentos da sociedade, envolvendo escolas, associações de bairro e comunidades carentes.
O quadro geral dos resultados expõe a necessidade de adaptação a um novo paradigma em formação, onde os diversos atores ainda não se aproximaram o bastante, para que os entraves detectados neste trabalho possam ser superados, permitindo um salto qualitativo no gerenciamento dos resíduos de serviços de saúde na área da Medicina Veterinária. Os dados sugerem a necessidade de um trabalho articulado e simultâneo de divulgação e conscientização em, pelo menos, duas frentes: a primeira direcionada aos profissionais médicos veterinários, abordando as implicações legais, ambientais, mercadológicas e sanitárias pertinentes ao gerenciamento dos resíduos de serviços de saúde e a segunda frente, voltada às instituições regulamentadoras e fiscalizadoras; às acadêmicas; ao setor privado e às organizações de classe, aproximando-as entre si e da rotina vivenciada nos estabelecimentos médicos veterinários, no sentido de perceber e corrigir as lacunas na formação profissional; na percepção dos riscos representados pelos resíduos de serviços de saúde; no diálogo entre as instituições e o profissional e na formulação de critérios que adequem a legislação pertinente às características e à escala da geração de resíduos de serviços de saúde nos estabelecimentos médicos veterinários.

\section{AGRADECIMENTOS}

Ao Conselho Nacional de Desenvolvimento Cientifico e Tecnológico $(\mathrm{CNPq})$ pelo auxílio financeiro recebido para o desenvolvimento desse trabalho. 
Rev. Bras. Saúde Prod. Anim., Salvador, v.14, n.2, p.287-298 abr./jun., 2013 http://www.rbspa.ufba.br ISSN 15199940

\section{REFERÊNCIAS}

BARROS, D.X. de; FRANCO, L.C.; TIPPLE, A.F.V.; BARBOSA, M.A; SOUZA, A.C.S. Exposição a material biológico no manejo externo dos resíduos de serviço de saúde. Cogitare Enfermagem, v.15 n.1 p.82-86, 2010.

BAUER, M.W. Análise de conteúdo clássica: uma revisão. In: BAUER, M.W.; GASKELL, G. Pesquisa qualitativa com texto, imagem e som: um manual prático. Petrópolis, RJ: Vozes; 2002 p.189-217.

BARBOSA, M.V.F.; GUIMARÃES, J.E.; ALMEIDA, M.A.O.; GONDIM, L.F.P.; REGIS, G.B. Freqüência de anticorpos IgG anti-Toxoplasma gondii em soros de cães errantes da cidade de Salvador-Bahia, Brasil. Brazilian Journal of Veterinary Research and Animal Science, v.40, n.6, p.457-465, 2003.

BARBOZA, D.C.P.M.; LEAL, D.C.; SOUZA, B.M.P.S.; CARNEIRO, A.J.B.; GOMES NETO, C.M.B.; ALCÂNATARA, A.C.; JULIÃO, F.S.; MOURA, S.A.B.; PERALVA, L.M.P.; FERREIRA, F.; FRANKE, C.R. Inquérito epidemiológico da leishmaniose visceral canina em três distritos sanitários do Município de Salvador, Bahia, Brasil. Revista Brasileira de Saúde e Produção Animal [online], v.10, n.2, p.434-447, 2009.

BELLAN, N.; PINTO, T.J.A.; KANEKO, T.M.; MORETTO, L.D.; SANTOS JUNIOR, N. dos.Critical analysis of the regulations regarding the disposal of medication waste. Brazilian Journal of Pharmaceutical Sciences, v.48, n.3, p.507-518, 2012.
BRASIL. Ministério da Saúde. Agência Nacional de Vigilância Sanitária. Resolução RDC no 306, de 07 de dezembro de 2004. Dispõe sobre o Regulamento Técnico para o gerenciamento de resíduos de serviços de saúde. Diário Oficial da União, Brasília, DF. 2004.

BRASIL. Ministério da Saúde. Agência Nacional de Vigilância Sanitária.

Manual de gerenciamento de resíduos de serviços de saúde Brasília, 2006.

BRASIL. Ministério da Saúde. Agência Nacional de Vigilância Sanitária.

Referencia Técnica para o Funcionamento dos Serviços Veterinários. Brasília, 2010.

BRASIL. CONAMA. Resolução No. 358. Dispõe sobre o tratamento e a disposição final dos resíduos dos serviços de saúde e dá outras providências. Diário Oficial da União, Brasília, DF. 2005.

CAVALCANTI, L.A.; DASSO, M.G.; OLIVEIRA, F.C.S.; VIEGAS, S.A.R.A.; ALMEIDA, M.G.A.R.; ANUNCIAÇÃO, A.V.M.; ALCANTARA, A.C.; BITTENCOURT, D.V.V.; OLIVEIRA, E.M.D.Pesquisa de anticorpos antibrucella canis em cães provenientes da região metropolitana de Salvador.

\section{Revista Brasileira de Saúde e} Produção Animal [online], v.7, n2, p.176-180, 2006.

CORRÊA, L.B.; LUNARDI, V.L.; CONTO, S.M. de; GALIAZZI, M.C. O saber resíduos sólidos de serviços de saúde na formação acadêmica: uma contribuição da educação ambiental. Interface Comunicação em Saúde, v.9, n.18, p.571-84, 2005. 
CONSELHO FEDERAL DE

MEDICINA VETERINÁRIA - CFMV.

Resolução do CFMV N.680/00.

Conceitua e estabelece condições de

funcionamento dos estabelecimentos

médicos veterinários. Brasília, 2000.

CONSELHO FEDERAL DE

MEDICINA VETERINÁRIA -

CFMV/BA. Manual de Orientação e

Procedimentos do Responsável

Técnico. 7ed. Bahia, 2007. .46p.

GARCIA, L.P. ; ZANETTI-RAMOS,

B.G. Gerenciamento dos resíduos de

serviços de saúde: uma questão de

biossegurança. Caderno de Saúde

Pública, v.20, n.3, p.744-752, 2004.

GONÇALVES, E.M.N; SANTOS,

C.B.S; BADARÓ, M.L.S;

FARIAS,V.A; RODRIGUES, E.;

MENDES, M.E; SUMITA,N.M.

Modelo de implantação de plano de

gerenciamento de resíduos no

laboratório clínico. Jornal Brasieliro

de Patologia e Medicina Laboratorial, v.47, n.3, p.249-255, 2011.

GOUVEIA, N. Resíduos sólidos urbanos: impactos socioambientais e perspectiva de manejo sustentável com inclusão social. Ciência \& saúde coletiva, v.17, n.6, p.1503-1510, 2012 .

MBONGWE, B.; MMEREKI, B.T.; MAGASHULA, A. Healthcare waste management: current practices in selected healthcare facilities, Botswana. Waste Management, v.28, n.1, p.226233, 2008.

NASCIMENTO, T.C; JANUZZI, W.A; LEONEL, M; SILVA; V.L.; DINIZ, C.G. Ocorrência de bactérias clinicamente relevantes nos resíduos de serviços de saúde em um aterro sanitário brasileiro e perfil de susceptibilidade a antimicrobianos.
Revista da Sociedade Brasileira de Medicina Tropical; v.42,n.4,p.415419, 2009.

NAZAR, M.W.; PORDEUS, I.A.; WERNECK, M. A. F. Gerenciamento de resíduos sólidos de odontologia em postos de saúde da rede municipal de Belo Horizonte, Brasil. Revista Panamericana de Salud Publica, v. 17, n.4,p.237-42,2005.

NOGUEIRA-MARTINS, M.C.F.; BOGUS, C.M. Considerações sobre a metodologia qualitativa como recurso para o estudo das ações de humanização em saúde. Saúde e Sociedade, v.13, n.3, p.44-57, 2004.

ORGANIZAÇÃO PAN-AMERICANA DA SAÚDE - OPAS.. Guia para o manejo interno de resíduos sólidos em estabelecimentos de saúde. Brasília, 1997.

PECHANSKY, F.; INCIARDI, J.S; SURRATT, H.; LIMA, A.F.B.S; KESSLER, F.P.; SOIBELMAN, M.; HIRAKATA,V. Estudo sobre as características de usuários de drogas injetáveis que buscam atendimento em Porto Alegre, RS. Revista Brasileira de Psiquiatria, v.22, p.164-71, 2000.

PILGER, R.R ;SCHENATO, F. Classificação dos resíduos de serviços de saúde de um hospital veterinário.

Engenharia Sanitária e Ambiental, v.13, n. 1 p.23-28, 2008.

REZENDE, L.R. Vulnerabilidade dos geradores de resíduos de saúde frente às Resoluções n. 358 CONAMA e RDC n. 306 ANVISA. O Mundo da Saúde, v.30 n.4, p.588-597,2006.

ROEDER-FERRARI, L.D.; ANDRIGUETTO FILHO, J.M.; FERRARI, M.V. Produção e manejo de 
resíduos sólidos de saúde no hospital veterinário da UFPR. Archives of Veterinary Science, v.13, n.1, p.26-30, 2008.

SALES,C.C. de L.;SPOLTI, G.; LOPES, M.S.B.; LOPES, D.F.

Gerenciamento dos resíduos sólidos dos serviços de saúde: aspectos do manejo interno no município de Marituba, Pará, Brasil. Ciência \& Saúde Coletiva, v.14, n.6, p.2231-2238, 2009.

SANTOS, N.M.; SILVA, V.M.G.; THÉ, T.S.; SANTOS, A.B.; SOUZA; T.P. Contaminação das praias por parasitos caninos de importância zoonótica na orla da parte alta da cidade de Salvador-Ba. Revista de Ciências Médicas e Biológicas, v.5, n.1, p.40-47, 2006.

SILVA, D.D. Plano de Gerenciamento de resíduos de Serviços de Saúde (PGRESÍDUOS DE SERVIÇOS DE SAÚDE ): Uma ferramenta eficaz na melhoria do desempenho ambiental das unidades de saúde. Opinio Verbis, v.2, n.2, p.69-83,2005.
VASCONCELLOS, E.A. de; FARIAS, M.S.S. de; DINIZ, M.J; MACIEL, J.L. Diagnóstico do problema dos residuos sólidos hospitalares: o caso de campina grande (PB). Revista Brasileira de Geografia Médica e da Saúde, v.2, n.3, p.28-34, 2006.

WORLD HEALTH ORGANIZATION - WHO. National health-care waste management plan guidance manual. To reduce the burden of disease, healthcare waste needs sound management, including alternatives to incineration. Switzerland, 2004.

Data de recebimento: 07/01/2013

Data de aprovação: 23/05/2013 